\title{
Avaliação da implantação da atenção à hipertensão arterial pelas equipes de Saúde da Família do município do Recife (PE, Brasil)
}

\author{
The implementation analysis of the arterial hypertension care \\ by the Family Health teams in Recife city (Pernambuco, Brazil)
}

Juliana Martins Barbosa da Silva Costa ${ }^{1}$

Maria Rejane Ferreira da Silva ${ }^{1}$

Eduardo Freese de Carvalho ${ }^{1}$

${ }^{1}$ Centro de Pesquisas Aggeu Magalhães, Fundação Oswaldo Cruz. Av. Moraes Rego $s / n^{\circ}$, Cidade Universitária. 50670-420 Recife PE.

jumarbs@cpqam.fiocruz.br

\begin{abstract}
The implementation analysis of the arterial hypertension care by the Family Health teams in Recife city (Pernambuco, Brazil) was based on a normative evaluation of the structure and work process and analysis of some context elements. An interview was applied to a randomized sample of doctors and nurses of 72 Family Health teams and the hypertension programme coordinator, and official documents were analyzed. A score system was used to classify the performance of the implementation level in excellent (when 90$100 \%$ of activities were implemented); adequate (70-79\%); inadequate (50-69\%); and critical $(<50 \%)$. The results show a significant weakness in the arterial hypertension care by the Family Health teams in Recife. The absence of specific programme coordination is related to the low performance observed. The implementation level was considered inadequate and some problems were identified due to the poor infrastructure, deficient inputs, low professional qualification for hypertension care and insipient use of information for planning the actions.
\end{abstract}

Key words Arterial hypertension, Health analysis, Basic attention, Family Health Program
Resumo A avaliação da atenção à hipertensão arterial pelas equipes de Saúde da Família do Recife (PE) foi realizada por meio de uma avaliação normativa da estrutura e do processo de trabalho e da análise de alguns elementos contextuais. Para tanto, foram entrevistados os médicos e enfermeiros de uma amostra aleatória representativa que abrangeu 72 equipes e os coordenadores do programa de hipertensão, além da análise de documentos oficiais. Para definição do grau de implantação, foi utilizado um sistema de escores que permitiu classificá-lo em excelente (90-100\% das atividades implantadas); satisfatório (70-79\%); insatisfatório (50-69\%); e crítico $(<50 \%)$. Foi detectada uma importante fragilidade na atenção ao hipertenso. A ausência de coordenação geral do programa de hipertensão no nível central e a insipiência das coordenações nos distritos sanitários contribuíram para o baixo desempenho encontrado. O grau de implantação foi considerado insatisfatório, sendo identificados problemas relativos à adequação da área física, deficiência de insumos, inadequada qualificação profissional na atenção ao hipertenso e um insipiente uso da informação para o planejamento das ações.

Palavras-chave Hipertensão, Avaliação em saúde, Atenção básica, Programa Saúde da Família 


\section{Introdução}

As intensas transformações políticas, econômicas e sociais por que vem passando o Brasil nas últimas décadas têm gerado complexos processos de transição demográfica, epidemiológica e nutricional ${ }^{1,2}$. As principais consequências dessas "transições" são o aumento na longevidade da população com um importante incremento na expectativa de vida, modificações nos hábitos alimentares e nos estilos de vida e a passagem de um perfil de elevada mortalidade em crianças, com predomínio das doenças infecciosas e parasitárias, para um perfil de elevada mortalidade em idades mais avançadas, provocadas por doenças crônicas não transmissíveis e por causas externas ${ }^{2-4}$.

Neste cenário, a hipertensão arterial se apresenta como um dos principais problemas de saúde pública da atualidade. Esse agravo se caracteriza por apresentar um curso clínico lento e assintomático, uma elevada prevalência, múltiplos fatores de risco que agem sinergicamente, dificuldades em seu controle e, quando não adequadamente tratada, um grande número de complicações, principalmente no sistema cardiovascular, que vêm se apresentando como a principal causa de óbito no país desde a década de $1960^{4,5}$.

Apesar da reconhecida possibilidade de prevenção de uma considerável parcela das doenças cardiovasculares por meio do controle adequado da pressão arterial e da adoção de hábitos de vida saudáveis, durante muitos anos não se observou por parte das instâncias dos governos federal, estaduais e municipais a adoção de políticas específicas e amplas medidas de intervenção que permitissem uma articulação de ações de prevenção, diagnóstico precoce, acompanhamento sistemático e tratamento dos hipertensos.

A primeira tentativa de implementação de uma política voltada para esse agravo ocorreu no final da década de 1980 . A organização das ações estava baseada na lógica dos programas de saúde de caráter vertical e centralizado no Ministério da Saúde, predominando as ações individuais e medicalizantes, não se conseguindo, desta forma, obter um impacto positivo na morbimortalidade por enfermidades cardiovasculares em nível populacional ${ }^{5,6}$.

Mesmo com uma baixa efetividade, esse programa norteou a política de prevenção e controle da hipertensão arterial por mais de dez anos. Apenas em 2001 foram estabelecidas novas diretrizes para orientação da atenção a esse agravo. Do ponto de vista normativo, duas medidas foram de grande importância: a promulgação da
Norma Operacional da Assistência à Saúde (NOAS) $01^{7}$, que estabeleceu as diretrizes para ampliação do acesso e da qualidade da atenção básica e definiu o controle da hipertensão arterial como área de atuação estratégica mínima desse nível de atenção, e o lançamento do Plano de Reorganização da Atenção à Hipertensão Arterial e ao Diabetes Mellitus ${ }^{8}$.

Além disso, a ampliação da cobertura dos serviços básicos de saúde proporcionada pela Estratégia Saúde da Família melhorou o acesso da população aos serviços básicos de saúde. Essa estratégia permitiu, entre outros avanços, a criação de vínculo entre os usuários e a Equipe de Saúde da Família, favorecendo um acompanhamento mais sistemático e a ampliação das atividades de promoção e prevenção9. Esses avanços vieram somar esforços à implantação das diretrizes propostas pelo Plano de Reorganização da Atenção à Hipertensão Arterial e ao Diabetes Mellitus ${ }^{8}$.

A concepção deste plano prevê a atualização dos profissionais da rede básica de saúde, a realização de ações de promoção da saúde e controle de fatores de risco, a garantia de diagnóstico e vinculação dos hipertensos às unidades básicas de saúde para tratamento e acompanhamento, a disponibilização contínua de medicamentos e a instituição de elencos mínimos de informações sobre a ocorrência desses agravos ${ }^{8}$.

Considerando os importantes avanços na normatização existente e a escassez de estudos que considerem a avaliação normativa do programa de hipertensão arterial, o presente estudo busca avaliar a implantação da atenção à hipertensão arterial pelas equipes de Saúde da Família do município do Recife (PE), identificando os principais entraves na implantação e execução das atividades propostas visando aperfeiçoá-las e melhorar a qualidade do serviço ofertado.

\section{Métodos}

Foi realizada uma avaliação normativa das dimensões da estrutura e do processo de trabalho e foram analisados alguns elementos do contexto político-normativo e técnico-gerencial e organizacional que possam ter influenciado o grau de implantação.

A área de estudo foi o município do Recife (PE). Segundo estimativa do Censo do Instituto Brasileiro de Geografia e Estatística (IBGE) ${ }^{10}$ para o ano de 2006, esse município possuía uma população de aproximadamente 1.500 .000 habitantes. Seu território está dividido em 94 bairros distribuídos em 
seis regiões político-administrativas (RPA). Cada RPA corresponde a um distrito sanitário, que é a unidade gerencial mínima da Secretaria Municipal de Saúde. Esse município está habilitado na gestão plena do sistema municipal e apresenta a Estratégia Saúde da Família como o eixo reorganizador da atenção básica. Em dezembro de 2005, possuía 103 Unidades de Saúde da Família com 213 equipes, o que representava uma cobertura de 44,95\% da população residente no município.

A realização do estudo ocorreu em cinco etapas: a primeira correspondeu à construção do modelo lógico da atenção ao hipertenso na atenção básica, por meio da análise dos documentos oficiais que tratam da atenção ao hipertenso ${ }^{7,8,11-}$ 15. Para a construção do modelo lógico, foram inicialmente identificados os problemas (ações de controle da hipertensão pouco efetivas, eleva- da prevalência de hipertensão e elevada morbimortalidade por doenças cardiovasculares). Após essa etapa, apontou-se a intervenção a ser avaliada (reorganização da atenção à hipertensão arterial, tendo como eixo a atenção básica), foram identificados os componentes da intervenção (ações de prevenção, de controle e o uso dos sistemas de informação) e subcomponentes (as ações de controle foram divididas em: diagnóstico, vinculação, tratamento e sistema de referência). Para cada componente e/ou subcomponente, foram especificados a estrutura necessária (recursos humanos e materiais) e o processo adequado (ações a serem realizadas), visando a resultados a curto e médio prazos que levariam a longo prazo à redução da morbimortalidade por hipertensão arterial e suas complicações cardiovasculares (Figura 1).

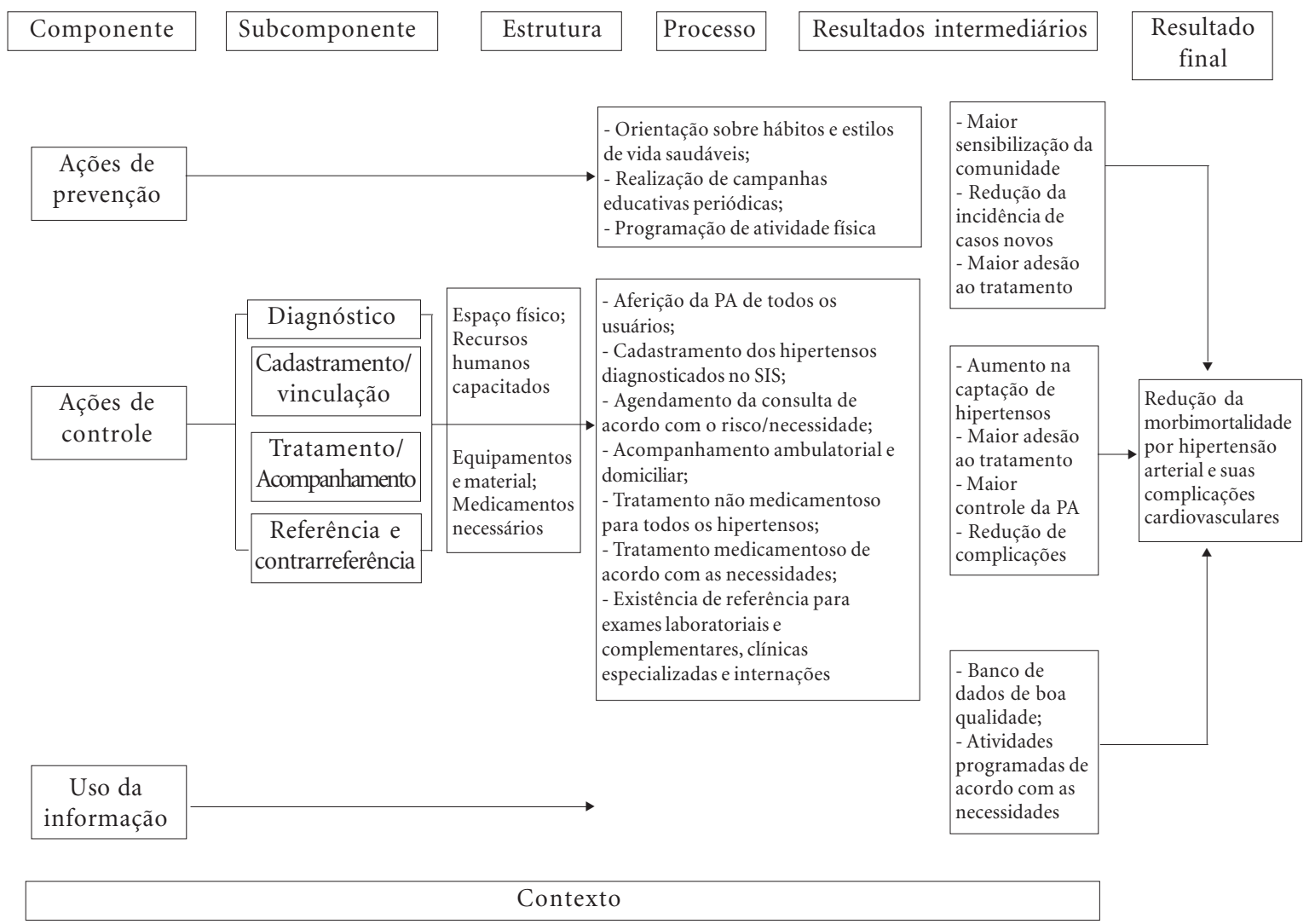

Figura 1. Modelo lógico da atenção à hipertensão arterial desenvolvida pelas equipes de Saúde da Família. 
O modelo lógico subsidiou a construção dos instrumentos de coleta de dados para as etapas seguintes que compreenderam: a avaliação da estrutura e do processo de trabalho, a determinação do grau de implantação e a análise de aspectos contextuais.

Para avaliação da estrutura e do processo de trabalho, utilizou-se um questionário estruturado aplicado aos médicos e enfermeiros de uma amostra representativa das equipes de Saúde da Família do município. No processo de amostragem considerou-se o total de equipes (213) que estavam prestando atividades assistenciais em dezembro de 2005. Foram utilizados os seguintes parâmetros: $\mathrm{p}=85 \%$, erro $10 \%$, efeito do desenho= 1,3 . A seleção das equipes de Saúde da Família ocorreu por sorteio após a definição de uma amostra de 72 equipes que correspondeu a um terço das equipes do município.

Para verificação do grau de implantação foi utilizado um sistema de escores, no qual cada item analisado recebeu uma pontuação. Esta foi atribuída de acordo com a relação de importância na atenção ao hipertenso, identificada através de revisão teórica sobre o assunto. Os itens referentes à estrutura receberam peso quatro, e ao processo, peso seis. A partir da pontuação máxima e da pontuação obtida, foi calculado por meio de uma regra de três simples o grau de implantação que foi por conveniência definido em: excelente, quando apresentou de $90-100 \%$ das atividades implantadas, satisfatório de 70-79\%, insatisfatório de 50-69\% e crítico <50\%.

Para análise do contexto foram entrevistados os coordenadores do programa de hipertensão nos distritos sanitários e foram analisados os Planos Municipais de Saúde 2002/2005 ${ }^{16}$ e $2006 / 2009^{17}$, nos quais procuraram-se identificar as propostas de priorização da hipertensão no município, bem como as ações de prevenção e controle da hipertensão realizadas e planejadas para os próximos anos.

\section{Resultados}

\section{Grau de implantação}

O grau de implantação da atenção à hipertensão arterial desenvolvida pelas equipes de Saúde da Família do Recife foi classificado como insatisfatório (58,8\%). Entre os distritos sanitários do município não foi observada uma variação significativa. Todos os distritos sanitários apresentaram um grau de implantação insatisfatório, com percentual de cumprimento das atividades preconizadas variando entre $54,1 \%$ e 65,0\% (Tabela 1).

Tanto a dimensão da estrutura quanto a do processo de trabalho foram classificadas como insatisfatórias. Contudo, a dimensão da estrutura apresentou características ainda mais inadequadas do que o processo de trabalho, aproximando-se do nível crítico do grau de implantação (Tabela 1).

\section{Avaliação da dimensão da estrutura}

No que se refere à dimensão da estrutura, foram avaliados os componentes: área física, recursos humanos, equipamentos e materiais essenciais e medicamentos essenciais (Figura 1). O componente da estrutura que apresentou o melhor desempenho foi o referente à distribuição de medicamentos essenciais para o controle da hipertensão com um grau de implantação satisfatório (Tabela 2). Apesar de quase totalidade das equipes de Saúde da Família disponibilizarem esses medicamentos, o que contribuiu para a pontuação final obtida, foi verificado que apenas 37,5\% das ESF possuíam regularidade em sua distribuição (Tabela 2).

A área física das Unidades de Saúde da Família apresentaram-se inadequadas para a atenção do hipertenso de acordo com os princípios e diretrizes do Programa de Saúde da Família e do

Tabela 1. Grau de implantação da atenção à hipertensão arterial pelas equipes de Saúde da Família segundo distritos sanitários - Recife (PE), 2006.

\begin{tabular}{lccccccc}
\hline \multicolumn{1}{c}{ Dimensões } & $\begin{array}{c}\text { DS I } \\
(\mathbf{N}=\mathbf{5})\end{array}$ & $\begin{array}{c}\text { DS II } \\
(\mathbf{N}=\mathbf{1 3})\end{array}$ & $\begin{array}{c}\text { DS III } \\
(\mathbf{N}=\mathbf{1 3})\end{array}$ & $\begin{array}{c}\text { DS IV } \\
(\mathbf{N}=\mathbf{1 1})\end{array}$ & $\begin{array}{c}\text { DS V } \\
(\mathbf{N}=\mathbf{9})\end{array}$ & $\begin{array}{c}\text { DS VI } \\
(\mathbf{N}=\mathbf{2 1})\end{array}$ & $\begin{array}{c}\text { Recife } \\
(\mathbf{N}=\mathbf{7 2})\end{array}$ \\
\hline Estrutura & 51,2 & 53,7 & 59,0 & 49,1 & 44,4 & 52,6 & $\mathbf{5 2 , 4}$ \\
Processo & 61,4 & 61,0 & 69,0 & 62,3 & 60,5 & 63,5 & $\mathbf{6 3 , 0}$ \\
Grau de implantação (estrutura x 4+ & $\mathbf{5 7 , 3}$ & $\mathbf{5 8 , 1}$ & $\mathbf{6 5 , 0}$ & $\mathbf{5 7 , 0}$ & $\mathbf{5 4 , 1}$ & $\mathbf{5 9 , 1}$ & $\mathbf{5 8 , 8}$ \\
processo x 6)/10 & & & & & & &
\end{tabular}

$\mathrm{N}=$ tamanho da amostra. 
Plano de Reorganização da Atenção à Hipertensão Arterial e ao Diabetes Mellitus. As principais limitações se referem ao número suficiente de consultórios para a consulta médica e de enfermagem e a existência de sala para a realização de palestras e reuniões, dificultando a realização de atividades educativas em grupos (Tabela 2).

Além dos problemas anteriormente citados, os insumos para a realização das ações de prevenção e controle da hipertensão arterial eram insuficientes, com um grau de implantação considerado crítico (Tabela 2). Grande parte das ESF não seguia um protocolo para o atendimento clínico dos hipertensos, não existia manutenção periódica dos equipamentos e os materiais educativos eram escassos (Tabela 2).

Associados a esses problemas relativos aos recursos materiais, os recursos humanos apresentaram uma inadequada qualificação dirigida à atenção ao hipertenso. Apesar de quase a totalidade dos médicos e enfermeiros ter realizado curso de especialização em Saúde Pública ou Saúde da Família, apenas um quinto dos profissionais realizaram capacitação específica na atenção ao hipertenso. Destaca-se ainda o fato de os profissionais de nível médio (auxiliar de enfermagem e agente comunitário de saúde) não terem recebido capacitação específica necessária para

Tabela 2. Componentes, itens, pontuação máxima, pontuação obtida, percentual de cumprimento das atividades e grau de implantação da dimensão da estrutura - Recife (PE), 2006.

\begin{tabular}{|c|c|c|c|c|c|}
\hline Componente & Item & $\begin{array}{c}\text { Pontuação } \\
\text { máxima }\end{array}$ & $\begin{array}{c}\text { Pontuação } \\
\text { obtida }\end{array}$ & $\%$ & Grau \\
\hline \multirow[t]{3}{*}{ Área física } & Sala de recepção & 2,0 & 1,94 & 97,2 & \multirow{3}{*}{$\begin{array}{c}55,6 \\
\text { insatisfatório }\end{array}$} \\
\hline & Sala de palestra/reunião & 3,0 & 1,33 & 44,4 & \\
\hline & Consultório em $\mathrm{n}^{\circ}$ suficiente & 3,0 & 1,17 & 38,9 & \\
\hline \multirow{10}{*}{$\begin{array}{l}\text { Recursos } \\
\text { humanos }\end{array}$} & Especialização Saúde da Família (médico) & 1,0 & 0,92 & 91,7 & \multirow{10}{*}{$\begin{array}{l}44,0 \\
\text { crítico }\end{array}$} \\
\hline & Especialização Saúde da Família (enfermeiro) & 1,0 & 1,00 & 100,0 & \\
\hline & Introdutório PSF (médico) & 0,5 & 0,35 & 69,4 & \\
\hline & Introdutório PSF (enfermeiro) & 0,5 & 0,39 & 77,8 & \\
\hline & Introdutório PSF (auxiliar de enfermagem) & 0,5 & 0,26 & 51,4 & \\
\hline & Introdutório PSF (ACS) & 0,5 & 0,35 & 69,4 & \\
\hline & Capacitação hipertensão (médico) & 1,5 & 0,56 & 37,5 & \\
\hline & Capacitação hipertensão (enfermeiro) & 1,5 & 0,38 & 25,0 & \\
\hline & $\begin{array}{l}\text { Capacitação hipertensão (auxiliar de } \\
\text { enfermagem) }\end{array}$ & 1,5 & 0,10 & 6,9 & \\
\hline & Capacitação hipertensão (ACS) & 1,5 & 0,10 & 6,9 & \\
\hline \multirow{11}{*}{$\begin{array}{l}\text { Equipamento } \\
\text { e materiais }\end{array}$} & Estetoscópio & 0,5 & 0,39 & 77,8 & \multirow{11}{*}{$\begin{array}{c}45,9 \\
\text { crítico }\end{array}$} \\
\hline & Tensiômetro & 1,0 & 0,81 & 80,6 & \\
\hline & Manguito $(12,16$ e 18$)$ & 0,5 & 0,04 & 8,3 & \\
\hline & Balança antropométrica & 0,5 & 0,42 & 84,7 & \\
\hline & Fita métrica & 0,5 & 0,46 & 91,7 & \\
\hline & Manutenção equipamentos & 2,0 & 0,11 & 5,6 & \\
\hline & Protocolo hipertensão & 1,05 & 0,38 & 25,0 & \\
\hline & Manual hipertensão & 1,0 & 0,43 & 43,1 & \\
\hline & Material educativo & 2,5 & 0,66 & 26,4 & \\
\hline & Ficha B SIAB & 1,0 & 0,86 & 86,1 & \\
\hline & Ficha Hiperdia & 1,0 & 0,96 & 95,8 & \\
\hline \multirow{5}{*}{$\begin{array}{l}\text { Medicamentos } \\
\text { essenciais }\end{array}$} & Hidroclorotiazida & 1,0 & 0,97 & 97,2 & \multirow{5}{*}{$\begin{array}{c}74,8 \\
\text { satisfatório }\end{array}$} \\
\hline & Propanolol & 1,0 & 0,97 & 97,2 & \\
\hline & Captopril & 1,0 & 0,97 & 97,2 & \\
\hline & Medicação urgência & 1,0 & 0,83 & 83,3 & \\
\hline & $\begin{array}{l}\text { Regularidade na distribuição de } \\
\text { medicamentos }\end{array}$ & 2,0 & 0,75 & 37,5 & \\
\hline
\end{tabular}


sua atuação no manejo e cuidado aos portadores de hipertensão (Tabela 2).

\section{Avaliação da dimensão do processo de trabalho}

Em relação à dimensão do processo de trabalho, foram avaliados os componentes: uso da informação, ações de prevenção e ações de controle. As ações de controle, por sua vez, estavam divididas em quatro subcomponentes: diagnóstico de casos, cadastramento (vinculação), tratamento/acompanhamento e sistema de referência (Figura 1).

O componente uso da informação foi classificado como crítico (Tabela 3). A adesão por parte dos profissionais na alimentação dos sistemas de informação, principalmente o SisHiperdia, foi baixa, comprometendo a qualidade das informações disponíveis. Além disso, o uso da informação para o planejamento de ações não fazia parte da rotina de grande parte das ESF estudadas (Tabela 3).

As ações de prevenção foram classificadas como insatisfatórias (Tabela 3). Todas as equipes de Saúde da Família realizavam algum tipo de atividade educativa, contudo predominaram as atividades individuais voltadas para os hipertensos como parte do tratamento. As ações de prevenção destinadas à população em geral visando à prevenção da hipertensão arterial e seus fatores de risco eram desenvolvidas apenas por uma pequena parcela das equipes de Saúde da Família (Tabela 3).

Em relação às ações de controle, 98,6\% das ESF realizavam o diagnóstico clínico da hipertensão arterial. A captação dos hipertensos era feita predominantemente por meio da aferição da pressão arterial dos usuários da unidade de Saúde da Família. Apenas 25,0\% das equipes utilizavam outras estratégias para captação de casos novos como a realização de campanhas e mutirões com a comunidade. Além disso, em menos da metade das equipes os profissionais estratificavam o risco cardiovascular dos hipertensos (Tabela 3).

Existiam cotas para a realização dos exames laboratoriais básicos na quase totalidade das equipes de Saúde da Família. Contudo, foram identificados problemas na qualidade dos exames e no tempo de entrega dos resultados. Problema semelhante foi detectado no encaminhamento dos hipertensos para consultas em clínicas especializadas. Apesar de a maioria das ESF possuírem referência para clínicas especializadas, a demora no agendamento das consultas comprometeu a qualidade da atenção ofertada. Além disso, apenas $10,0 \%$ das ESF afirmou possuir unidades hospitalares de maior complexidade como referência (Tabela 3 ).

\section{Análise do contexto}

Em relação ao contexto, considerando sua dimensão político-normativa, foram identificados alguns avanços quanto ao reconhecimento da hipertensão arterial como um problema de saúde prioritário no município. $\mathrm{Na}$ análise do Plano Municipal de Saúde do Recife do período de 2002 a 2005, observou-se que não existia um planejamento de ações voltadas para o controle da hipertensão arterial. Contudo, na análise do Plano Municipal de Saúde do período seguinte (2006 a 2009), identificou-se a hipertensão arterial como um dos principais problemas de saúde do município. Além disso, foram estabelecidas proposições e metas, tanto para ações de promoção e prevenção quanto para assistência e vigilância desse agravo.

No que se refere à dimensão técnico-gerencial e organizacional, os principais achados demonstraram que a ausência de uma coordenação geral do programa no município e a insipiência das coordenações nos distritos sanitários se apresenta como um dos principais entraves na operacionalização da atenção ao hipertenso.

Os responsáveis pela coordenação nos distritos sanitários não recebiam apoio técnico e financeiro da instância central, ficando suas atividades restritas a ações burocráticas como o monitoramento do envio das fichas de cadastramento e acompanhamento do Hiperdia pelas unidades básicas de saúde, sem haver grandes exigências quanto à qualidade dos dados e à dispensação de medicamentos. Além desse fato, o uso da informação para o planejamento de ações não fazia parte da rotina da coordenação do programa de hipertensão nos distritos sanitários. Em nenhum distrito foi realizada análise da situação de saúde dos hipertensos ou a programação de ações voltadas para os portadores desta enfermidade.

\section{Discussão}

Nos últimos anos, a avaliação de programas e serviços de saúde vem ganhando relevância no cenário nacional. O Ministério da Saúde vem destinando maior volume de recursos na tentativa de promover a institucionalização da avalia- 
ção ${ }^{18}$, e tem-se verificado maior interesse por parte da academia em desenvolver estudos avaliativos. Apesar desse inegável avanço, a quantidade de estudos ainda é pequena, principalmente no que se refere à avaliação de programas vol- tados ao controle das doenças crônicas não transmissíveis, como a hipertensão arterial. A realização de pesquisas envolvendo essa problemática deveria se apresentar como prioritária na tentativa de equacionar uma série de problemas sani-

Tabela 3. Componentes, itens, pontuação máxima, pontuação obtida, percentual de cumprimento das atividades e grau de implantação da dimensão do processo de trabalho - Recife (PE), 2006.

\begin{tabular}{|c|c|c|c|c|}
\hline Componente & Item & $\begin{array}{l}\text { Pontuação } \\
\text { máxima }\end{array}$ & $\begin{array}{c}\text { DS VI } \\
(\mathrm{N}=21)\end{array}$ & $\begin{array}{r}\text { Recife } \\
(\mathrm{N}=72)\end{array}$ \\
\hline \multirow[t]{6}{*}{ Ações de prevenção } & Ações educativas & 0,5 & 52,6 & 52,4 \\
\hline & Campanhas & 2,0 & 63,5 & 63,0 \\
\hline & Palestras & 1,5 & 59,1 & 58,8 \\
\hline & Grupos de hipertensos & 2,5 & & \\
\hline & Hipertensos individualmente & 1,5 & & \\
\hline & Todos os profissionais envolvidos & 1,0 & & \\
\hline \multirow{6}{*}{$\begin{array}{c}\text { Diagnósticos } \\
\text { de casos }\end{array}$} & Diagnóstico clínico & 1,0 & & \\
\hline & Critério diagnóstico & 2,0 & & \\
\hline & Aferição da PA usuários da USF & 1,5 & & \\
\hline & Aferição PA gestantes & 1,5 & & \\
\hline & Aferição PA domicílio & 1,5 & & \\
\hline & Mutirões & 1,5 & & \\
\hline \multirow{8}{*}{$\begin{array}{l}\text { Cadastramento } \\
\text { e vinculação }\end{array}$} & Cadastra SIAB & 1,5 & & \\
\hline & Cadastra Hiperdia & 1,5 & & \\
\hline & Prontuário individualizado & 1,5 & & \\
\hline & Agendamento - risco & 2,5 & & \\
\hline & Frequência alto risco & 1,0 & & \\
\hline & Frequência médio risco & 1,0 & & \\
\hline & Frequência baixo risco & 1,0 & & \\
\hline & Ambulatorial & 1,0 & & \\
\hline & Domiciliar & 1,0 & & \\
\hline \multirow[t]{14}{*}{ e acompanhamento } & Tratamento não medicamentoso & 1,0 & & \\
\hline & O que consiste este tratamento & 1,0 & & \\
\hline & Tratamento medicamentoso & 2,0 & & \\
\hline & Acompanha domicílio AVC & 1,0 & & \\
\hline & Frequência de acompanhamento & 1,0 & & \\
\hline & Glicemia capilar & 1,0 & & \\
\hline & Frequência da glicemia capilar & 1,0 & & \\
\hline & Atendimento de urgência & 1,0 & & \\
\hline & Domicílio após urgência & 1,0 & & \\
\hline & Busca ativa de faltosos & 1,5 & & \\
\hline & Envolvimento multiprofissional & 1,0 & & \\
\hline & Parceria intersetorial & 1,5 & & \\
\hline & Cota de exames & 1,0 & & \\
\hline & Exames laboratoriais & 1,5 & & \\
\hline \multirow[t]{6}{*}{ Sistema de referência } & Eletrocardiograma & 1,5 & & \\
\hline & RX tórax & 1,5 & & \\
\hline & Clínicas especializadas & 2,5 & & \\
\hline & Unidade hospitalar & 2,0 & & \\
\hline & Alimenta SIAB & 1,5 & & \\
\hline & Frequência alimentação SIAB & 1,5 & & \\
\hline \multirow[t]{3}{*}{ Uso da informação } & Alimenta Hiperdia & 2,0 & & \\
\hline & Frequência alimentação Hiperdia & 2,0 & & \\
\hline & Usa informação planejamento & 3,0 & & \\
\hline
\end{tabular}


tários advindos dos processos de transição demográfica, epidemiológica e nutricional pelo qual vem passando o Brasil nas últimas décadas ${ }^{1,2-4}$.

O presente estudo possibilitou identificar uma série de fragilidades na atenção ao hipertenso, apesar de as ações de prevenção e controle dessa enfermidade possuirem uma grande normatização por parte do Ministério da Saúde , $^{7,11-15}$. A ausência de uma coordenação geral do programa e a insipiência das coordenações nos distritos sanitários se apresentaram como um dos principais entraves na operacionalização das ações, na medida em que não havia uma articulação entre as ações preconizadas e as efetivamente realizadas pelas ESF. Embora, atualmente, a hipertensão seja considerada um dos principais problemas de saúde do município segundo o Plano Municipal de Saúde (2006 a 2009) ${ }^{16}$, ainda foram identificadas no primeiro ano de execução desse plano dificuldades por parte da gestão em assumir efetivamente esse problema como uma prioridade.

Como reflexo dessa desarticulação e da falta de priorização, a atenção à hipertensão arterial pelas equipes de Saúde da Família do Recife apresentou um grau de implantação insatisfatório. O percentual de cumprimento das atividades preconizadas pelos documentos oficiais foi de $58,8 \%$ - resultado inferior ao encontrado por Cavalcante $^{19}$ em Camaragibe (PE), que considerou o programa de controle da hipertensão como implantado e encontrou um percentual de cumprimento das atividades preconizadas de $85,8 \%$. Diferenças contextuais entre os municípios analisados podem explicar parcialmente os valores encontrados, além de diferenças metodológicas entre os estudos.

Entre os distritos sanitários não foram encontradas variações significativas no grau de implantação. Todos apresentaram um grau considerado insatisfatório. Os distritos sanitários compartilhavam as mesmas dificuldades que envolviam problemas de infraestrutura, deficiência de insumos, inadequada qualificação profissional na atenção ao hipertenso e insipiência na coordenação do programa de hipertensão.

Em relação à infraestrutura, as unidades básicas de saúde apresentaram-se inadequadas ao funcionamento das equipes de Saúde da Família, com um número insuficiente de consultório e ausência de sala para a realização de palestras e reuniões, dificultando a realização de algumas atividades propostas pelo Ministério da Saúde ${ }^{20}$. Essa inadequação no espaço físico é reflexo da precária readequação da rede básica de saúde ocorrida com a implantação do Programa de
Saúde da Família9, no qual muitas unidades de saúde tradicionais foram transformadas em unidades de Saúde da Família, sem que tenha havido reformas na infraestrutura, necessárias ao adequado funcionamento das equipes.

Outro aspecto da estrutura considerado crítico foi a disponibilidade de insumos para a realização das ações de prevenção e controle, comprometendo a qualidade do serviço ofertado. Entre esses se destacaram a ausência de manutenção periódica dos equipamentos e a disponibilidade de material educativo. Tais achados estão em conformidade com os encontrados em uma avaliação realizada pelo Ministério da Saúde que identificou a disponibilidade de material educativo em aproximadamente um quarto das equipes de Saúde da Família implantadas no Brasil em $1999^{18}$ e contrastam com os achados da pesquisa realizada por Cavalcante ${ }^{19}$, que encontrou material educativo em cerca de três quartos das equipes de Saúde da Família de Camaragibe (PE).

Além desses entraves, a ausência de um protocolo clínico no âmbito do município tem contribuído para o baixo desempenho apresentado na medida em que as ações de prevenção e controle não possuem uma diretriz específica e dependem da formação e informação de cada profissional. Considerando a complexidade no cuidado aos hipertensos, a dificuldade em se conseguir o controle ideal da pressão arterial com um único agente terapêutico e a necessidade de capacitação contínua dos profissionais ${ }^{21}$, a instituição de um protocolo de atendimento clínico se faz necessário no sentido de garantir uma prescrição segura e eficaz.

Somada à ausência de uma diretriz específica para a atenção aos hipertensos, a qualificação dos profissionais na atenção à hipertensão arterial apresentou-se inadequada. Apenas um terço dos profissionais realizou capacitação específica na atenção ao hipertenso. Esse quantitativo de profissionais capacitados mostrou-se inferior à média nacional, que em 2004 era de aproximadamente $50,0 \%{ }^{22}$.

Outra questão de grande importância para se obter um efetivo controle dos níveis tensionais e reduzir as complicações e comorbidades associadas à hipertensão arterial é a disponibilização ininterrupta de medicação anti-hipertensiva ${ }^{23}$. A quase totalidade das equipes estudadas afirmou disponibilizar medicação anti-hipertensiva. Contudo, a regularidade na distribuição mostrou-se inadequada em cerca de dois terços das equipes de Saúde da Família. Apesar de a disponibilidade dos medicamentos para os hipertensos estar assegu- 
rada pelo Programa Nacional de Assistência Farmacêutica ao Hipertenso ${ }^{13}$, problemas no cadastramento dos hipertensos têm impossibilitado um planejamento adequado, ocorrendo falta de medicação em várias unidades de Saúde da Família.

Os problemas organizacionais somados às limitações na estrutura (recursos humanos e materiais) influenciaram no desenvolvimento de algumas atividades do processo de trabalho (atividades de prevenção, controle e uso da informação) que apresentaram um grau de implantação insatisfatório.

O uso da informação para o planejamento de ações visando ao controle da hipertensão foi aspecto considerado crítico. Essa atividade não fazia parte da rotina da coordenação do programa nem dos profissionais das equipes de Saúde da Família. Apenas um quarto das equipes estudadas utilizava a informação para o planejamento das ações. Este resultado é inferior ao encontrado em avaliação realizada pelo Ministério da Saúde que identificou o uso da informação em cerca da metade das equipes de Saúde da Família do país ${ }^{23}$. Entretanto, os resultados são semelhantes aos achados de Sousa et al. ${ }^{24}$, que identificaram um elevado despreparo das equipes de Saúde da Família na utilização dos sistemas de informações para o planejamento e a avaliação das ações de saúde realizadas em sua área de abrangência.

No que se refere às ações de prevenção, estas são consideradas atividades estratégicas prioritárias do PSF e atribuição de todos os membros que integram a equipe de Saúde da Família9 . Apesar desse fato, o componente ações preventivas obteve um grau de implantação insatisfatório. Embora todas as ESF realizassem algum tipo de ação educativa, as atividades individuais predominaram em detrimento das atividades coletivas, ocorrendo uma baixa adesão das equipes às ações preventivas destinadas à população em geral. Estes achados estão em conformidade com os estudos de Noronha ${ }^{25}$ e Alves e Franco ${ }^{26}$, que demonstraram uma frequência reduzida no desenvolvimento de ações educativas coletivas pelas equipes de Saúde da Família.

No intuito de prevenir complicações e comorbidades, se faz necessária a captação precoce dos hipertensos para que sejam instituídas as medidas terapêuticas apropriadas a cada situação. Nesse sentido, o presente estudo identificou que esta atividade era realizada predominantemente por meio da aferição da pressão arterial dos usuários da Unidade de Saúde da Família; apenas um pequeno número de equipes realizava a busca ativa de hipertensos assintomáticos na comu- nidade. A insuficiência desse tipo de abordagem, focada apenas na demanda espontânea, foi criticada por Sala et al. ${ }^{27}$. Estes autores chamam a atenção para o fato de que uma parcela da população que não frequenta o serviço de saúde ou o faz em condições de atendimento muito sumárias não terá acesso a um diagnóstico precoce da hipertensão arterial. Dessa forma, torna-se necessária a adoção de abordagens alternativas em que as equipes de Saúde da Família entrem em maior contato com a comunidade, organizando atividades de triagem periódicas para a detecção de casos suspeitos.

No que se refere ao tratamento dos hipertensos, a Sociedade Brasileira de Hipertensão ${ }^{28}$ recomenda a estratificação do risco cardiovascular do indivíduo para a prescrição do tratamento mais adequado, seja ele medicamentoso ou não. O presente estudo identificou que apenas a metade das equipes realizava essa atividade. A ausência de um protocolo de atendimento clínico no município e a inadequada qualificação dos profissionais na atenção ao hipertenso podem ter contribuído para o pequeno percentual de seguimento dessa recomendação.

Apesar da expressiva ampliação da oferta dos serviços básicos de saúde com a implantação do Programa de Saúde da Família, o acesso aos serviços de média e alta complexidades ainda se constitui em um verdadeiro entrave a um atendimento integral ao hipertenso. Em relação ao sistema de referência, foi observada uma certa organização dos serviços de média complexidade que estão sob gestão municipal. Existiam cotas para a realização de exames laboratoriais básicos e para clínicas especializadas na quase totalidade das equipes estudadas. Contudo, um terço dos entrevistados afirmou que as cotas para a realização dos exames laboratoriais eram insuficientes, e muitos dos profissionais identificaram ainda como entraves a demora no recebimento dos resultados e a qualidade dos exames. Além disso, foi observada uma demanda maior do que a oferta para as clínicas especializadas, principalmente para o cardiologista. Considerando que os indivíduos que eram referenciados para este profissional eram os casos de maior gravidade, a integralidade da atenção e sua resolutividade encontravam-se comprometidas.

Embora se tenha observado uma certa organização do sistema de referência para os serviços de média complexidade, a referência para unidades hospitalares de alta complexidade era praticamente inexistente. A maioria dos hospitais de grande porte de Pernambuco se encontra no território do 
Recife; contudo, essas unidades hospitalares se encontram sob gestão estadual, apesar de o município estar habilitado em gestão plena do sistema municipal de saúde. Dessa forma, torna-se necessária maior articulação entre as instâncias gestoras estadual e municipal no intuito de se implementar um sistema de referência e contrarreferência que venha contribuir para a efetivação da integralidade da atenção oferecida à população.

\section{Colaboradores}

JMBS Costa, MRF Silva e EF Carvalho participaram de todas as etapas de elaboração do artigo.

\section{Agradecimentos}

Os autores agradecem ao Conselho Nacional de Desenvolvimento Científico e Tecnológico (CNPq) o financiamento do Projeto Avaliação da Implantação da Atenção à Hipertensão Arterial pelas Equipes de Saúde da Família do Município do Recife-PE.
Pelo exposto, e por ser a hipertensão arterial uma enfermidade crônica para a qual não existe cura, mas com a possibilidade de prevenção e de controle efetivo, a adoção de medidas e ações adequadas de vigilância à saúde pelas ESF podem reduzir o impacto socioeconômico provocados por este agravo e contribuir para a melhoria na qualidade de vida da população.

\section{Referências}

1. Freese E, Fontbonne A. Transição epidemiológica comparada: modernidade, precariedade e vulnerabilidade. In: Freese E, organizador. Epidemiologia, políticas e determinantes das doenças crônicas não transmissíveis no Brasil. Recife: Ed. Universitária da UFPE; 2006. p. 17-46.

2. Cesse E, Freese E. Características e determinantes do padrão brasileiro de ocorrência das DCNT no século XX. In: Freese E, organizador. Epidemiologia, políticas e determinantes das doenças crônicas não transmissíveis no Brasil. Recife: Ed. Universitária da UFPE; 2006. p. 47-72.

3. Kalanche A. O envelhecimento da população mundial: um desafio novo. Rev Saude Publica 1987; 21(3):200-210.

4. Laurenti R. Transição demográfica e transição epidemiológica. In: Anais do I Congresso Brasileiro de Epidemiologia. Campinas; 1990. p. 143-165.

5. Lessa I. Doenças crônicas não transmissíveis. In: Lessa I. O adulto brasileiro e as doenças da modernidade: epidemiologia das doenças crônicas não-transmissíveis. São Paulo: Hucitec; Rio de Janeiro: Abrasco; 1998. p. 29-42.

6. Lessa I. Doenças crônicas não transmissíveis no Brasil: um desafio para a complexa tarefa de vigilância. Cien Saude Colet 2004; 9(4):931-943.

7. Brasil. Gestão Municipal de Saúde: leis, normas e portarias atuais. Portaria no 95/GM, de 26 de janeiro de 2001. Rio de Janeiro: Ministério da Saúde; 2001.

8. Brasil. Ministério da Saúde. Plano de reorganização da atenção à hipertensão arterial e ao Diabetes mellitus. Brasília: Ministério da Saúde; 2001.

9. Brasil. Ministério da Saúde. Saúde da Família: uma estratégia para a reorientação do modelo assistencial. Brasília: Ministério da Saúde; 1997.

10. Instituto Brasileiro de Geografia e Estatística (IBGE). Censo Demográfico 2000. Rio de Janeiro: IBGE; 2001. p. 11-23.

11. Brasil. Portaria conjunta $n^{\circ} 2$, de 5 de março de 2002. Implantação do Plano de Reorganização da Atenção à Hipertensão Arterial e ao Diabetes Mellitus. Brasília; 2002. [acessado 2005 out 07]. Disponível em: http://dtr2001.saude.gov.br/sas/cnhd/legislacao/ port $2002 /$ port $2 . h$ tm 
12. Brasil. Portaria no $235 / \mathrm{GM}$, de 20 de fevereiro de 2001. Que estabelece as diretrizes para a reorganização da atenção aos segmentos populacionais expostos e portadores de hipertensão arterial. Brasília; 2001. [acessado 2004 set 9]. Disponível em: http:// dtr2001saude.gov.br/sas/cnhd/legislacao/port2001/ port 235

13. Brasil. Portaria no 371/GM, de 4 de março de 2002. Institui o Programa Nacional de Assistência Farmacêutica para Hipertensão Arterial e Diabetes Mellitus. Brasília; 2002. [acessado 2005 out 7]. Disponível em: htttp://dtr2001.saude.gov.br/sas/cnhd/legislacao/ port2002/port371.htm

14. Brasil. Ministério da Saúde. Manual de hipertensão arterial e diabetes mellitus. Brasília: Ministério da Saúde; 2001.

15. Brasil. Portaria no 648, de 28 de março de 2006. Aprova a Política Nacional de Atenção Básica, estabelecendo a revisão de diretrizes e normas para a organização da Atenção Básica para o Programa Saúde da Família (PSF) e o Programa Agentes Comunitários de Saúde (PACS). Diário Oficial da República Federativa do Brasil 2006; 28 mar.

16. Pernambuco. Recife. Plano Municipal de Saúde 20022005. Recife: Secretaria Municipal de Saúde do Recife; 2002.

17. Pernambuco. Recife. Plano Municipal de Saúde 20062009. Recife: Secretaria Municipal de Saúde do Recife; 2006.

18. Felisberto E. Da teoria à formulação de uma política nacional de avaliação: reabrindo o debate. Cien Saude Colet 2006; 11(3):553-563.

19. Cavalcante MGS. Avaliação da implantação das áreas estratégicas mínimas da atenção básica nas equipes de Saúde da Família de Camaragibe-PE, 2004 (dissertação). Recife: Instituto Materno-Infantil de Pernambuco; 2004.

20. Brasil. Ministério da Saúde. Avaliação da implantação e funcionamento do Programa de Saúde da Família: análise por estados; 2001. [acessado 2005 maio 31]. Disponível em: http://www.saude.gov.br/ publicacoes/psf
21. Zaitune MPA, Barros MBA, César CLG, Carandina L, Goldbaum M. Hipertensão arterial em idosos: prevalência, fatores associados e práticas de controle no município de Campinas, São Paulo, Brasil. Cad Saude Publica 2006; 22(2):285-294.

22. Brasil. Ministério da Saúde. Avaliação normativa do Programa de Saúde da Família no Brasil: monitoramento da implantação e funcionamento das Equipes de Saúde da Família - 2001/2002. Brasília: Ministério da Saúde; 2004.

23. Piccini RX, Facchini LA, Tomasi E, Thumé E, Silveira DS, Siqueira FV, Rodrigues MA. Necessidades de saúde comuns aos idosos: efetividade na oferta e utilização em atenção básica à saúde. Cien Saude Colet 2006; 11(3):657-667.

24. Sousa LB, Souza RKT, Scochi MJ. Hipertensão arterial e Saúde da Família: atenção aos portadores em município de pequeno porte na região sul do Brasil. Arq Bras Cardiol 2006; 87(1):496-503.

25. Noronha CV. Projeto Saúde para Todos na América Latina: identificando as necessidades de capacitação dos profissionais do Programa Saúde da Família. Salvador: Ed. UFBA; 2004.

26. Alves VS, Franco ALS. Estratégias comunicacionais do médico de Saúde da Família para educação em saúde no contexto clínico. Cien Saude Colet 2003; 8(1):144-149.

27. Sala A, Cohen DD, Dalmaso ASW, Lima AMM, Teixeira RR. Metodologia de avaliação do trabalho na atenção à saúde. Cad Saude Publica 1998; 14(4):741-751.

28. Sociedade Brasileira de Cardiologia, Sociedade Brasileira de Hipertensão, Sociedade Brasileira de Nefrologia, organizadores. V Diretrizes Brasileiras de Hipertensão. São Paulo: SBC, SBH, SBN; 2006. [acessado 2006 nov 3]. Disponível em: http://bvsms. saude.gov.br/bvs/publicacoes/v_diretrizes_brasileira_ hipertensao_arterial_2006.pdf

Artigo apresentado em 28/06/2007

Aprovado em 13/12/2007

Versão final apresentada em 13/01/2008 